\title{
Article \\ Spread Mechanism and Control Strategies of Rumor Propagation Model Considering Rumor Refutation and Information Feedback in Emergency Management
}

\author{
Jianhong Chen, Chaoqun Chen, Qinghua Song, Yifei Zhao $\mathbb{B}$, Longxin Deng, Raoqing Xie and Shan Yang * \\ School of Resources and Safety Engineering, Central South University, Changsha 410083, China; \\ cjh@263.net (J.C.); cchaoqun@163.com (C.C.); csuluck0110@csu.edu.cn (Q.S.); zhaoyifei@csu.edu.cn (Y.Z.); \\ 15179099633@163.com (L.D.); raoqing.xie@163.com (R.X.) \\ * Correspondence: yangshan@csu.edu.cn
}

Citation: Chen, J.; Chen, C.; Song, Q.; Zhao, Y.; Deng, L.; Xie, R.; Yang, S. Spread Mechanism and Control Strategies of Rumor Propagation Model Considering Rumor Refutation and Information Feedback in Emergency Management. Symmetry 2021, 13, 1694. https://doi.org/ $10.3390 /$ sym 13091694

Academic Editors: Yongwimon Lenbury, Elvin Moore and Ravi P. Agarwal

Received: 17 August 2021

Accepted: 13 September 2021

Published: 14 September 2021

Publisher's Note: MDPI stays neutral with regard to jurisdictional claims in published maps and institutional affiliations.

Copyright: (c) 2021 by the authors. Licensee MDPI, Basel, Switzerland. This article is an open access article distributed under the terms and conditions of the Creative Commons Attribution (CC BY) license (https:// creativecommons.org/licenses/by/ $4.0 /)$.

\begin{abstract}
The rumor-free equilibrium state and rumor-endemic equilibrium state are two symmetric descriptions of the status of a system. The constant spreading of rumors would affect the smooth operation of emergency management procedures and cause unnecessary social and economic loss. To reduce the negative effect of rumor propagation, in this paper, we introduce a compartmental model of rumor propagation, which considers the rumor refutation of public and information feedback. By deriving mean-field equations that describe the dynamics of the model, we use analytical and numerical solutions of these equations to investigate the threshold and dynamics of the model in both the closed system and open system. The results imply that the initial equilibrium point is not stable and there exists a rumor-free equilibrium point; in the open system, there exists a threshold beyond which rumors can spread; the stability of the initial equilibrium point is related to the threshold $\mathrm{R}_{0}=\left(\varphi^{*} \alpha\right) / \mu$, and there exists a rumor-endemic equilibrium point. The development process of rumor propagation can be divided into four stages: latent period, progressive period, intense period, and recession period. Under the influence of population, rumor spreading can exceed the threshold readily because the migration rate $\mu$ is usually less than the proportion of ignorants without critical ability $\varphi$, and the rumor spreading process in an open system presents a fluctuating development, the rumor would not disappear in this autonomous system. Based on the analysis, we propose some measures, such as providing open and efficient information queries and exchange platforms, etc.
\end{abstract}

Keywords: rumor propagation; emergency management; control strategies; information feedback; critical consciousness

\section{Introduction}

A rumor about iodized salt that can protect against radiation has spread rapidly throughout China since the Fukushima nuclear power plant exploded after Japan's earthquake occurred on 11 March 2011. The rumor triggered a panic purchase behavior of iodized salt. Large crowds doubted the rumor refutation information and persisted in buying salt, leading to social and economic chaos. Due to the suddenness, urgency, high uncertainty, and destructiveness of an emergent event (such as an earthquake), if the comprehensive and accurate information cannot be timely spread to people, the formation and propagation of rumors can be caused. In emergency management, the process of rumor propagation is also a process of negative emotion diffusion. Rumors can mislead people to make irrational behaviors, hinder the smooth progress of emergency management, and expand the losses triggered by the emergent events. As a consequence, rumor spreading control is a crucial issue in emergency management.

An army of scholars investigated the rules of rumor propagation by constructing propagation models from different dimensions, among which the Maki and Thomson (DK) [1] model and the Maki and Thomson (MT) [2] model are two classical rumor propagation 
models. In these classical models, people are subdivided into three groups: ignorants, spreaders, and stiflers. The ignorants refer to people who are not aware of rumor information, the spreaders refer to people who are spreading rumor information, and the stiflers refer to people who have heard the rumor but do not propagate it anymore. The transformation mechanism between different groups is as follows: the spreader-ignorant contact will convert the ignorant into the spreader, spreader-spreader contact will convert both spreaders into stiflers since both spreaders lose interest in spreading rumor information, and spreader-stifler contact will stifle the spreader. The MT model differs from the DK model in the termination mechanism of rumor spreading, namely, when a spreader and another spreader contact in the MT model, only the initial spreader becomes the stifler.

A series of modified rumor propagation models have been developed based on the classical models mentioned above. On the one hand, some researchers focused on the fundamental processes involved in rumor propagation. They introduced the social reinforcement mechanism [3], hesitation mechanism [4], etc. It's worth noting that time delay [5-7] may also influence rumor propagation. Huo et al. [8] believed that when the spreader contacts the ignorant, the ignorant individual first experiences an incubation period before turning into a spreader. Time delay not only affects the crowds, but also expert intervention and government policy [9], the effect of delay made an important contribution to the stability of the system. Moreover, some researchers redefined and redivided the groups in the rumor propagation model. Wang et al. [10] argued that there are rationals who can verify the rumor in the initial state of the rumor spreading system. With the exception of rationals, the incubators and wise individuals were introduced by Huo et al. [3,8]; the counterattack group was introduced by Zan et al. [11]; the exposed individuals by Xia et al. [12]. In addition, considering the rumor propagation system has close contact with the outside world, some scholars take the population migration into account $[6,7,13]$. They first assumed that the rumor propagation system has constant immigration and emigration, then studied the impact of other influencing factors, such as delayed on the rumor spreading and aimed to figure out the stability of the equilibrium point and the final condition of rumor spreading.

On the other hand, many scholars explored the rumor propagation model from the perspective of individuals and the rumor itself. Huo et al. [14] reckoned that the transmission rate of ignorant to the spreader is affected by psychological effects in the context of emergencies. In addition, the forgetting mechanism and the remembering mechanism of crowds were considered [4,15-17]. Wang et al. [18] claimed that whether the ignorants trust the spreaders or not determines the conversion rate of ignorants into spreaders after the contact of spreaders and ignorants. Moreover, the transmission rate is higher when the spreaders are credible. Similarly, Qiu et al. [19] found that a spreader with a high reputation can promote the spread of rumors. Furthermore, the education rate is a significant factor that may affect rumor propagation. Afassinou [20] built the Spreader, Educated Ignorant, Non-educated Ignorant, Stiflers (SEIR) rumor spreading model, and explored the impact of the education rate on rumor propagation. In this rumor propagation model, ignorants were distinguished by educated ignorants and non-educated ignorants. The educated ignorants have a lower probability of becoming spreaders and a higher probability of becoming stiflers after contacting the spreaders. Nevertheless, the uneducated ignorants have a higher probability of turning into spreaders and a lower probability of turning into stiflers after contacting spreaders. Additionally, in a like manner, Hui et al. [21] concluded that the higher-educators class is more likely to be immune to rumors with a higher probability and the lower-educators class is more inclined to accept and spread the rumors. With the development of information science, rumors have altered their spread mode to Online Social Networks (OSNs); hence, Hosni et al. [22] investigated the effect of OSN addiction on rumor propagation. They found that addicted individuals may accelerate the dissemination under specific conditions. As for the rumor itself, Chen et al. [23] suggested that rumor credibility and the correlation between rumor and people's lives will influence the dissemination of rumors. The higher the credibility of a rumor is, the larger the final scale of 
rumor spreading is, and the rumor propagation speed and spreading scale are proportional to the relevance between a rumor and people's lives. Xia et al. [12] maintained that when rumors had high attractiveness, the exposed individuals would decide to spread rumors and become the spreader; when the rumor was fuzzy, spreaders would cease spreading rumors and transform into exposed individuals; when the fuzziness of rumors was low, the spreaders would lose interest in spreading rumors and transform into stiflers. Moreover, when it comes to eradicating rumors, rumor intensity will contribute to the identification of the stop condition for anti-rumor propagation and the initial spreaders of anti-rumors [24].

Typically, numerous scholars investigated the relationship between rumor spreading and emergency management as well as developed the corresponding interplay model. Zhang et al. [25] found that rumor spreading has the effect of restricting the rapid expansion of the impact of emergencies. Huo et al. [26] stated that in emergencies, the official responses to emergencies produce positive social utility, while the negative social utility brought by the spread of rumors weaken the positive social utility. The authorities can take actions to control the spread of rumors to downplay negative social utility. However, if the emergency response is not enough to achieve the expected effect of the public, the negative social utility will be maximized. Zhao et al. [27] stated that the authorities can control the situation by using trusted media outlets with high audience ratings to disseminate information.

Existing research mainly focused on the definition, causes, influencing factors, and the final spreading state, but neglected to give a reasonable description of the development process of rumors. In addition, though some works of literature considered the educational level of individuals $[3,21,22]$, an individual's ability to resist rumor spreading is not fully considered. People's critical ability to refute rumors does not just pertain to theoretical knowledge acquired through education, but also many other factors, such as conscious behavior [3]. Individuals with critical ability can obtain the evidence of false rumor information through effective ways (i.e., rumor refutation information) and can further suppress the rumor propagation through effective feedback and rumor refutation information. Furthermore, in the era of the internet, people are connected under one virtual society, people on the network migrate frequently, namely, people can easily participate in a discussion of a topic, as conduct the spreading of rumors. Hence, the impact of population migration on rumor propagation should be comprehensively analyzed. However, in the very few studies that incorporate population migration, in most cases, population migration is served as a prerequisite to study the influence of other influencing factors on the spread of rumors. However, the influence of population migration on rumor spreading has not been studied in depth. Based on the above analysis, this paper establishes a new compartmental model of rumor propagation, and the main contributions of this paper can be summarized as follows:

(1) The rumor refutation mechanism and information feedback mechanism are considered. The groups in the rumor propagation system are redivided and redefined, a new kind of people - the skeptic is introduced into the model, and the stiflers are divided into stiflers who believe the rumor and stiflers who do not believe the rumor.

(2) The rumor propagation model is analyzed in both the closed system and open system, and the development process of rumor spreading is comprehensively described, and the general rules of rumor propagation under the influence of population migration are studied.

(3) Multiple influencing factors besides effective feedback mechanisms are comprehensively considered in this paper.

The rest of the article is structured as follows: In Section 2, the rumor propagation model in a closed system and an open system are established, the group division in the rumor propagation model and the behavior law of each group are analyzed, the mean-field equations are established. In Section 3, the general rules of rumor propagation and the rumor propagation laws under different influencing factors of a closed system are analyzed through the data obtained numerically. In Section 4, the general rules of rumor propagation 
and the rumor propagation laws under different influencing factors of an open system were analyzed through the data obtained numerically. Finally, Section 5 summarizes the paper.

\section{Rumor Propagation Models Considering Rumor Refutation and Information Feedback}

From the perspective of managing the public sentiment crisis in emergency management, the population in the rumor propagation system was subdivided into five groups in accordance with the rumor propagation process: ignorants $(X)$, spreaders $(Y)$, skeptics $(W)$, stiflers who believed the rumor $\left(Z_{1}\right)$, and stiflers who did not believe the rumor $\left(Z_{2}\right)$. In emergency management, public panic is easily caused by rumor spreading. The negative impact scale of rumors can be described as the proportion of stiflers who believe the rumor at the equilibrium state of rumor spreading. Then, ignorants were divided into two groups on the ground of whether they possess critical ability: ignorants with critical ability and ignorants without critical ability. Additionally, the proportion between the two groups was constant in the initial state of a specific rumor spreading model. Different ignorants had diverse behavioral responses to rumors. Ignorants with critical ability could not easily accept rumors and would become skeptics after contacting the spreaders. However, ignorants without critical ability accepted rumors readily and became spreaders after contacting the spreaders. Skeptics did not spread rumor information and actively obtained the refutation information. If refutation information was not available, skeptics became stiflers who believed the rumor; if refutation information was available, skeptics became stiflers who did not believe the rumor. When spreaders contacted other spreaders or stiflers who believed the rumor, they found that the rumor information was obsolete and stopped spreading the rumor information, and became stiflers who believed the rumor. While spreaders contacted stiflers who did not believe the rumor, the latter would persuade the spreaders to become stiflers who did not believe the rumor under the effective rumor refutation information feedback. Lastly, in terms of the method of Maki [2], it was thought that a rumor propagated when a spreader directly contacted others [28]. Additionally, each spreader completed a random contact process within a time step, and the skeptic took at least a time step to obtain the refutation information.

\subsection{Rumour Propagation Model XYWZ1Z2-C in a Closed System}

In this part, a closed rumor propagation system which has no population immigration and emigration was considered. Additionally, the rumor propagation model XYWZ1Z2-C in a closed system was established, as shown in Figure 1.

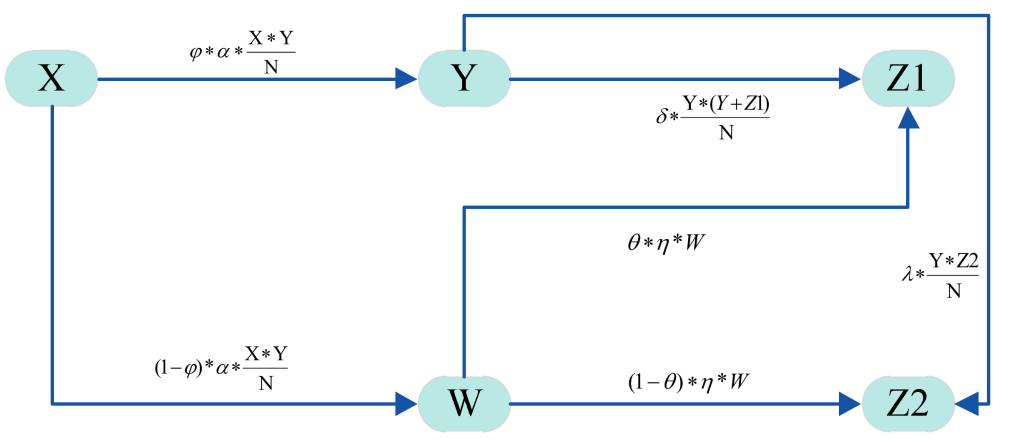

Figure 1. Rumor propagation model XYWZ1Z2-C in a closed system considering rumor refutation information feedback mechanism. 
According to the mean-field theory, the mean-field equations of the rumor propagation model XYWZ1Z2-C in a closed system were derived as follows:

$$
\left\{\begin{array}{l}
\frac{d X}{d t}=-\alpha * \frac{X * Y}{N} \\
\frac{d Y}{d t}=\varphi * \alpha * \frac{X * Y}{N}-\delta * \frac{Y *(Y+Z 1)}{N}-\lambda * \frac{Y * Z 2}{N}, \\
\frac{d W}{d t}=(1-\varphi) * \alpha * \frac{X * Y}{N}-\eta * W \\
\frac{d Z 1}{d t}=\delta * \frac{Y *(Y+Z 1)}{N}+\theta * \eta * W \\
\frac{d Z 2}{d t}=(1-\theta) * \eta * W+\lambda * \frac{Y * Z 2}{N} .
\end{array}\right.
$$

The parameters in the mean-field equations of rumor propagation were defined as follows: $N$ refers to the total population in the rumor propagation system; $X, Y, W, Z_{1}$, and $Z_{2}$, respectively, represent the population of ignorants, spreaders, skeptics, stiflers who believe the rumor, and stiflers who do not believe the rumor; $\varphi$ denotes the proportion of ignorants without critical ability in all ignorants; $(1-\varphi)$ represents the proportion of ignorants with critical ability in all ignorants; $\alpha$ refers to the conversion rate of the ignorants into the spreaders or the skeptics when the ignorants contact the spreaders; $\delta$ is the conversion rate of the first spreader into the stifler who believes the rumor when the spreader contacts other spreaders or stiflers who believe the rumor; $\eta$ is the rate of the skeptics change into stiflers who believe the rumor or stiflers who do not believe the rumor, which is related to the importance of rumor information identification; $\theta$ represents the ratio of the conversion rate of the skeptics into stiflers who believe the rumor to the total conversion rate of the skeptics; $(1-\theta)$ represents the ratio of the conversion rate of the skeptics into stiflers who do not believe the rumor to the total conversion rate of the skeptics, which is related to rumor discrimination capability; $\lambda$ is the conversion rate of the spreaders into stiflers who do not believe the rumor when contacting the stiflers who do not believe the rumor, which indicates the intensity of the rumor refutation information feedback mechanism.

Let $x=X / N, y=Y / N, w=W / N, z_{1}=Z_{1} / N, z_{2}=Z_{2} / N$, and $x, y, w, z_{1}, z_{2}$, respectively, represent the population density of ignorants, spreaders, skeptics, stiflers who believe the rumor, and stiflers who do not believe the rumor in the rumor dissemination system. Then, Equation (1) can be expressed as:

$$
\left\{\begin{array}{l}
\frac{d x}{d t}=-\alpha * x * y \\
\frac{d y}{d t}=\varphi * \alpha * x * y-\delta * y *(y+z 1)-\lambda * y * z 2 \\
\frac{d w}{d t}=(1-\varphi) * \alpha * x * y-\eta * w \\
\frac{d z 1}{d t}=\delta * y *(y+z 1)+\theta * \eta * w \\
\frac{d z 2}{d t}=(1-\theta) * \eta * w+\lambda * y * z 2
\end{array}\right.
$$

Equation (2) should satisfy the constraint condition: $x+y+w+z_{1}+z_{2}=1$. To obtain the equilibrium point of Equation (2), the right side of Equation (2) should be equal to zero according to the definition of the equilibrium point of the differential equation, that is:

$$
\left\{\begin{array}{l}
\frac{d x}{d t}=f_{1}(x, y, w, z 1, z 2)=-\alpha * x * y=0 \\
\frac{d y}{d t}=f_{2}(x, y, w, z 1, z 2)=\varphi * \alpha * x * y-\delta * y *(y+z 1)-\lambda * y * z 2=0, \\
\frac{d w}{d t}=f_{3}(x, y, w, z 1, z 2)=(1-\varphi) * \alpha * x * y-\eta * w=0 \\
\frac{d z 1}{d t}=f_{4}(x, y, w, z 1, z 2)=\delta * y *(y+z 1)+\theta * \eta * w=0 \\
\frac{d z 2}{d t}=f_{5}(x, y, w, z 1, z 2)=(1-\theta) * \eta * w+\lambda * y * z 2=0 .
\end{array}\right.
$$


By solving Equation (3), we can obtain:

$$
\left\{\begin{array}{l}
x=x^{*} \\
y=0 \\
w=0 \\
z 1=z 1^{*} \\
z 2=z 2^{*}
\end{array}\right.
$$

where $x^{*}, z_{1}^{*}, z_{2}^{*}$ are positive values satisfying the constraint condition $x^{*}+z_{1}^{*}+z_{2}^{*}=1$. According to Equation (2), the dissemination and developing process of the rumor spreading system has no concern with the independent variable, i.e., time parameter. Thus, the rumor spreading system is autonomous, and the rumor spreading process is deterministic. When a spreader appears in the system, the rumor spreading process starts and eventually reaches the equilibrium state. At the equilibrium point, $y=0, w=0$, namely, there are no spreaders and skeptics in the system. Therefore, the system finally reaches the rumor-free equilibrium state, corresponding to the rumor-free equilibrium point $E^{*}=\left(x^{*}, 0,0, z_{1}^{*}, z_{2}^{*}\right)$.

The system is in the initial equilibrium state when there is no spreader in the system, where $x^{*}=1, z_{1}^{*}=0, z_{2}{ }^{*}=0$. Let the equilibrium point corresponding to this equilibrium state be the initial equilibrium point $\mathrm{E}_{0}=(1,0,0,0,0)$. Subsequently, the Jacobian matrix of Equation (2) was established to analyze the stability of the initial equilibrium point

$$
J=\left|\begin{array}{ccccc}
-\alpha * y & -\alpha * x & 0 & 0 & 0 \\
\varphi * \alpha * y & a_{22} & 0 & -\delta * y & -\lambda * y \\
(1-\varphi) * \alpha * y & (1-\varphi) * \alpha * x & -\eta & 0 & 0 \\
0 & 2 * \delta * y+\delta * z 1 & \theta * \eta & \delta * y & 0 \\
0 & \lambda * z 2 & (1-\theta) * \eta & 0 & \lambda * y
\end{array}\right|
$$

where $a_{22}=\varphi * \alpha * x-2 * \delta * y-\delta * z 1-\lambda * z 2$.

The Jacobian matrix of the system at the initial equilibrium point $E_{0}=(1,0,0,0,0)$ is:

$$
J_{0}=\left|\begin{array}{ccccc}
0 & -\alpha & 0 & 0 & 0 \\
0 & \varphi * \alpha & 0 & 0 & 0 \\
0 & (1-\varphi) * \alpha & -\eta & 0 & 0 \\
0 & 0 & \theta * \eta & 0 & 0 \\
0 & 0 & (1-\theta) * \eta & 0 & 0
\end{array}\right|
$$

The characteristic equation is:

$$
\begin{aligned}
\left|J_{0}-v * I\right| & =\left|\begin{array}{ccccc}
-v & -\alpha & 0 & 0 & 0 \\
0 & \varphi * \alpha-v & 0 & 0 & 0 \\
0 & (1-\varphi) * \alpha & -\eta-v & 0 & 0 \\
0 & 0 & \theta * \eta & -v & 0 \\
0 & 0 & (1-\theta) * \eta & 0 & -v
\end{array}\right| \\
& =\quad-1 * v^{3} *(v+\eta) *(v-\varphi * \alpha)=0 .
\end{aligned}
$$

In the above characteristic equation, there is a characteristic root $\left(\varphi^{*} \alpha\right)$, which is a positive real part. Consequently, the system is unstable at the initial equilibrium point $\mathrm{E}_{0}$. When being disturbed at the initial equilibrium point, the system is far away from the equilibrium state until there is no spreader or skeptic in the system. In other words, the rumor spreading ends, and the system reaches a new equilibrium state. We can imply that the closed rumor propagation model XYWZ1Z2-C does not have critical point leading to rumor propagation. Provided that a spreader appears in the system, it will inevitably lead to the spread of rumors in a certain range; thus, yielding some negative effects. 


\subsection{Rumor Propagation Model XYWZ1Z2-O in an Open System}

With the advent of OSNs, people are connected under one virtual society [24]. On a practical level, the rumor propagation system has close contact with the outside world. Hence, the population in each group could have changed. Therefore, there was a stable immigration rate and emigration rate in the rumor propagation system [6]. Then, an open system rumor spreading model XYWZ1Z2-O was established, as shown in Figure 2.

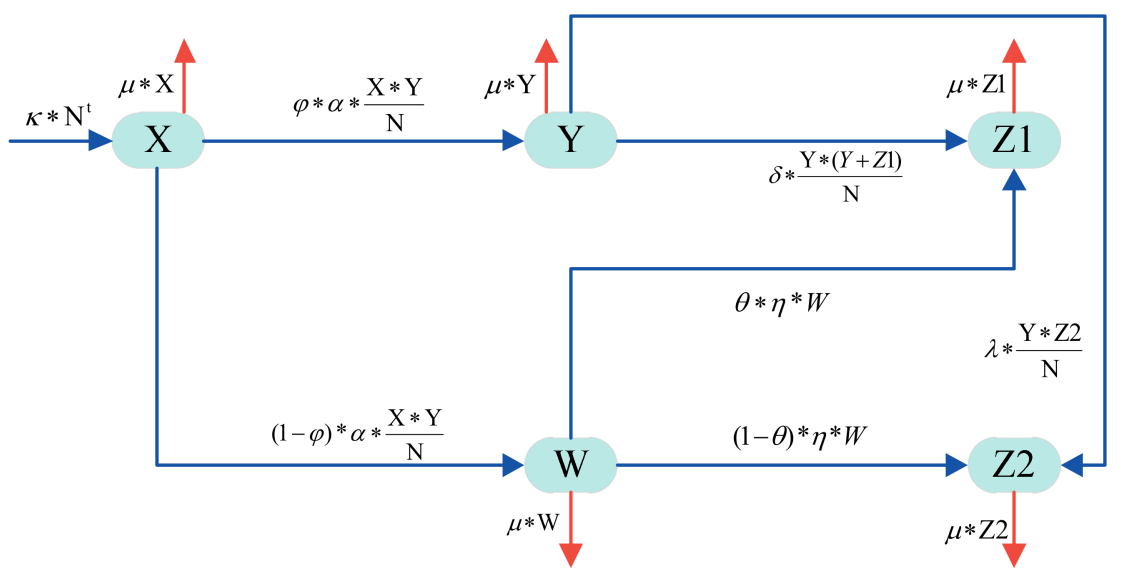

Figure 2. Rumor propagation model XYWZ1Z2-O in an open system considering rumor refutation information feedback mechanism.

The rumor spreading law of XYWZ1Z2-O can be expressed by mean-field equations as follows:

$$
\left\{\begin{array}{l}
\frac{d X}{d t}=\kappa * N^{t}-\alpha * \frac{X * Y}{N}-\mu * X, \\
\frac{d Y}{d t}=\varphi * \alpha * \frac{X * Y}{N}-\delta * \frac{Y *(Y+Z 1)}{N}-\lambda * \frac{Y * Z 2}{N}-\mu * Y, \\
\frac{d W}{d t}=(1-\varphi) * \alpha * \frac{X * Y}{N}-\eta * W-\mu * W, \\
\frac{d Z 1}{d t}=\delta * \frac{Y *(Y+Z 1)}{N}+\theta * \eta * W-\mu * Z 1, \\
\frac{d Z 2}{d t}=(1-\theta) * \eta * W+\lambda * \frac{Y * Z 2}{N}-\mu * Z 2 .
\end{array}\right.
$$

Equation (5) follow the parameter definition in Equation (2), and the new parameters are defined as follows: $N^{t}$ refers to the total population of the system at the $t$ time step; $\kappa$ represents the immigration rate of the rumor propagation system, and $\mu$ represents the emigration rate of the rumor propagation system. To simplify the rumor spreading model, we assumed that the immigration rate was equal to the emigration rate $[6-8,13]$, namely, $\kappa=\mu$. From Equation (5), the total population of the rumor propagation system did not change over time, that is $N^{t}=N$. Let $x=X / N, y=Y / N, w=W / N, z_{1}=Z_{1} / N, z_{2}=Z_{2} / N$, and $x, y, w, z_{1}, z_{2}$, respectively, represent the population density of ignorants, spreaders, skeptics, stiflers who believe the rumor, and stiflers who do not believe the rumor in the rumor spreading system. Given that $\tau=\mu t, \alpha_{0}=\alpha / \mu, \delta_{0}=\delta / \mu, \lambda_{0}=\lambda / \mu, \eta_{0}=\eta / \mu$, then Equation (5) can be transformed into:

$$
\left\{\begin{array}{l}
\frac{d x}{d \tau}=1-\alpha^{0} * x * y-x \\
\frac{d y}{d \tau}=\varphi * \alpha^{0} * x * y-\delta^{0} * y *(y+z 1)-\lambda^{0} * y * z 2-y, \\
\frac{d w}{d \tau}=(1-\varphi) * \alpha^{0} * x * y-\eta^{0} * w-w \\
\frac{d z 1}{d \tau}=\delta^{0} * y *(y+z 1)+\theta * \eta^{0} * w-z 1 \\
\frac{d z 2}{d \tau}=(1-\theta) * \eta^{0} * w+\lambda^{0} * y * z 2-z 2 .
\end{array}\right.
$$


To attain the equilibrium point of the system, let the right side of Equation (6) be equal to zero, and Equation (7) is obtained:

$$
\left\{\begin{array}{l}
\frac{d x}{d \tau}=f_{1}(x, y, w, z 1, z 2)=1-\alpha^{0} * x * y-x=0, \\
\frac{d y}{d \tau}=f_{2}(x, y, w, z 1, z 2)=\varphi * \alpha^{0} * x * y-\delta^{0} * y *(y+z 1)-\lambda^{0} * y * z 2-y=0, \\
\frac{d w}{d \tau}=f_{3}(x, y, w, z 1, z 2)=(1-\varphi) * \alpha^{0} * x * y-\eta^{0} * w-w=0, \\
\frac{d z 1}{d \tau}=f_{4}(x, y, w, z 1, z 2)=\delta^{0} * y *(y+z 1)+\theta * \eta^{0} * w-z 1=0, \\
\frac{d z 2}{d \tau}=f_{5}(x, y, w, z 1, z 2)=(1-\theta) * \eta^{0} * w+\lambda^{0} * y * z 2-z 2=0 .
\end{array}\right.
$$

From the first equation of Equation (7), we can obtain:

$$
x=\frac{1}{\alpha^{0} * y+1} .
$$

Inserting Equation (8) into the third equation of Equation (7), we can obtain:

$$
w=\frac{(1-\varphi) * \alpha^{0} * y}{\left(\eta^{0}+1\right) *\left(\alpha^{0} * y+1\right)} .
$$

Inserting Equations (8) and (9) into the fourth equation of Equation (7), we can obtain:

$$
z 1=\frac{\delta^{0} * y * y}{1-\delta^{0} * y}+\frac{\theta * \eta^{0}}{1-\delta^{0} * y} * \frac{(1-\varphi) * \alpha^{0} * y}{\left(\eta^{0}+1\right) *\left(\alpha^{0} * y+1\right)} .
$$

Inserting Equations (8)-(10) into the second equation of Equation (7), we can obtain:

$$
y *\left(a * y^{2}+b * y+c\right)=0 .
$$

And:

$$
\left\{\begin{array}{l}
a=\lambda^{0} * \alpha^{0} *\left(1+\delta^{0}\right)=\frac{\lambda * \alpha *(\mu+\delta)}{\mu * \mu * \mu}, \\
c=\varphi * \alpha^{0}-1=\frac{\varphi * \alpha-\mu}{\mu} .
\end{array}\right.
$$

Equation (11) has a constant zero solution, corresponding to the rumor-free propagation equilibrium point $\mathrm{E}_{\mathrm{F}}=(1,0,0,0,0)$. According to Equation (12), it was found that $a>0$; when $\varphi>\mu / \alpha, c<0$, a positive solution was added for Equation (11), corresponding to the rumor propagation equilibrium point $\mathrm{E}_{\mathrm{E}}=\left(x_{\mathrm{E}}, y_{\mathrm{E}}, w_{\mathrm{E}}, z_{1 \mathrm{E}}, z_{2 \mathrm{E}}\right)$. Given that $\mathrm{R}_{0}=\left(\varphi^{*} \alpha\right) / \mu$, if $R_{0}>1$, the system had a rumor-free equilibrium point and a rumor-endemic equilibrium point; if $R_{0} \leq 1$, the system only had a rumor-free equilibrium point and did not have a rumor-endemic equilibrium point.

The rumor-free equilibrium point $\mathrm{E}_{\mathrm{F}}$ took the same value as the initial equilibrium point $\mathrm{E}_{0}=(1,0,0,0,0)$. The stability of the system at the initial equilibrium point was analyzed below, and at the outset, the Jacobian matrix of Equation (6) was obtained:

$$
J=\left|\begin{array}{ccccc}
-\alpha^{0} * y-1 & -\alpha^{0} * x & 0 & 0 & 0 \\
\varphi * \alpha^{0} * y & a_{22} & 0 & -\delta^{0} * y & -\lambda^{0} * y \\
(1-\varphi) * \alpha^{0} * y & (1-\varphi) * \alpha^{0} * x & -\eta^{0}-1 & 0 & 0 \\
0 & 2 * \delta^{0} * y+\delta^{0} * z 1 & \theta * \eta^{0} & \delta^{0} * y-1 & 0 \\
0 & \lambda^{0} * z 2 & (1-\theta) * \eta^{0} & 0 & \lambda^{0} * y-1
\end{array}\right|
$$

where $a_{22}=\varphi * \alpha^{0} * x-2 * \delta^{0} * y-\delta^{0} * z 1-\lambda^{0} * z 2-1$. 
After solving the Jacobian matrix of Equation (6) at the initial equilibrium point $\mathrm{E}_{0}, J_{0}$ was obtained as follows:

$$
J_{0}=\left|\begin{array}{ccccc}
-1 & -\alpha^{0} & 0 & 0 & 0 \\
0 & \varphi * \alpha^{0}-1 & 0 & 0 & 0 \\
0 & (1-\varphi) * \alpha^{0} & -\eta^{0}-1 & 0 & 0 \\
0 & 0 & \theta * \eta^{0} & -1 & 0 \\
0 & 0 & (1-\theta) * \eta^{0} & 0 & -1
\end{array}\right|
$$

Then, the characteristic equation was obtained:

$$
\begin{aligned}
&\left|J_{0}-v * I\right|=\left|\begin{array}{ccccc}
-1-v & -\alpha^{0} & 0 & 0 & 0 \\
0 & \varphi * \alpha^{0}-1-v & 0 & 0 & 0 \\
0 & (1-\varphi) * \alpha^{0} & -\eta^{0}-1-v & 0 & 0 \\
0 & 0 & \theta * \eta^{0} & -1-v & 0 \\
0 & 0 & (1-\theta) * \eta^{0} & 0 & -1-v
\end{array}\right| \\
&= \\
&-1 *(v+1)^{3} *\left(v+\eta^{0}+1\right) *\left(v-\varphi * \alpha^{0}+1\right)=0 .
\end{aligned}
$$

It was simple to figure out that the characteristic roots of the characteristic equation were $-1,-1,-1,\left(-1-\eta_{0}\right),\left(-1+\varphi^{*} \alpha_{0}\right)$, i.e., $-1,-1,-1,(-1-\eta / \mu),\left(-1+\varphi^{*} \alpha / \mu\right)$. If $\varphi>\mu / \alpha$, i.e., $R_{0}>1$, all characteristic roots had strictly negative real parts, and the system was asymptotically stable at the initial equilibrium point $\mathrm{E}_{0}$. Since a spreader appeared in the system, the system deviated from the equilibrium state and, subsequently, returned to the initial equilibrium state. If $\varphi \leq \mu / \alpha$, i.e., $\mathrm{R}_{0} \leq 1$, the characteristic root had a positive real part; accordingly, the system was unstable at the initial equilibrium point $\mathrm{E}_{0}$. The system was far from the equilibrium state after the spreader appeared in the system. Therefore, if the proportion of ignorants without critical ability in the rumor spreading system was less than a certain value, the system could not reach the threshold point $R_{0}$ of rumor spreading, and rumors could not continue to spread and yield continuous negative influence.

\section{Analysis of Rumor Propagation Rules in a Closed System}

The development process of rumor propagation was related to multiple influencing factors in line with the rumor propagation model. The rumor propagation under general circumstances and the impact of changing in a single influencing factor on rumor propagation were analyzed below.

\subsection{Analysis of the General Rules of Rumor Propagation in a Closed System}

Given that the total population in the closed rumor spreading system was $N=10^{6}$, when a spreader appeared in the system at the initial state of rumor spreading, i.e., $Y=1$, and $X=N-1 \approx 10^{6}, W=0, Z_{1}=0, Z_{2}=0$. To analyze the general rules of rumor spreading in the closed rumor spreading system, the optimized parameters in Equation (1) were set (see Table 1). The four-order Runge-Kutta method [17,29] was used to solve Equation (1). The value of each group in each time step was recorded, and the developing trend of group density over time was plotted (see Figure 3).

Table 1. Parameter set for rumor propagation general rules analysis.

\begin{tabular}{cccccc}
\hline $\boldsymbol{\varphi}$ & $\alpha$ & $\delta$ & $\boldsymbol{\theta}$ & $\boldsymbol{\lambda}$ & $\lambda$ \\
\hline 0.5 & 0.2 & 0.2 & 0.1 & 0.5 & 0.2 \\
\hline
\end{tabular}

As illustrated in Figure 3, when a rumor spreader appeared in the system, the rumor propagated rapidly after an incubation period, then entered the stage of intense rumors spreading, and, afterward, entered the recession period. Ultimately, when the spreaders and skeptics in the rumor spreading system decreased to 0 , the rumor spreading was terminated, and the system reached the rumor-free equilibrium state. The development process of rumor dissemination can be divided into four stages: latent period, progressive 
period, intense period, and recession period. In the latent period, the ignorants came into contact with rumor information at a low rate, and the changes in each group were not obvious. In the progressive period, the ignorants accessed rumors at a faster rate and turned into spreaders or skeptics, and a significant trend of change could be observed in groups. In the intense period, the number of spreaders and skeptics reached a peak value, and the ignorant contacted with rumor information at a higher rate. In the recession period, the number of spreaders and skeptics gradually reduced and the speed of ignorant contact with rumors decreased. Finally, only ignorants, stiflers who believed the rumor and stiflers who did not believe the rumor existed in the system. The population density of stiflers who believed the rumor in the system could be employed to indicate the negative influence scale of rumor spreading. The decreased quantity of the population density of the ignorant, which was equal to the sum of the population density of stiflers who believed the rumor and stiflers who did not believe the rumor, could be utilized to evaluate the scope of rumor spreading. Additionally, rumor spreading control measures were primarily used to reduce the negative impact of the spread of rumors. Typically, the rumor propagation system shows the characteristic of symmetry, namely, the system is in the rumor-free equilibrium state in the initial stages, but the system is unstable at the initial equilibrium point. When rumors started to spread, the system would be far away from the equilibrium state, but, finally, the system reached a new equilibrium state. Additionally, if a spreader appeared in the system, the system would change to the rumor-endemic equilibrium state. The initial and final states of the system were the same. Moreover, the group density curves of spreaders also showed the same characteristic.

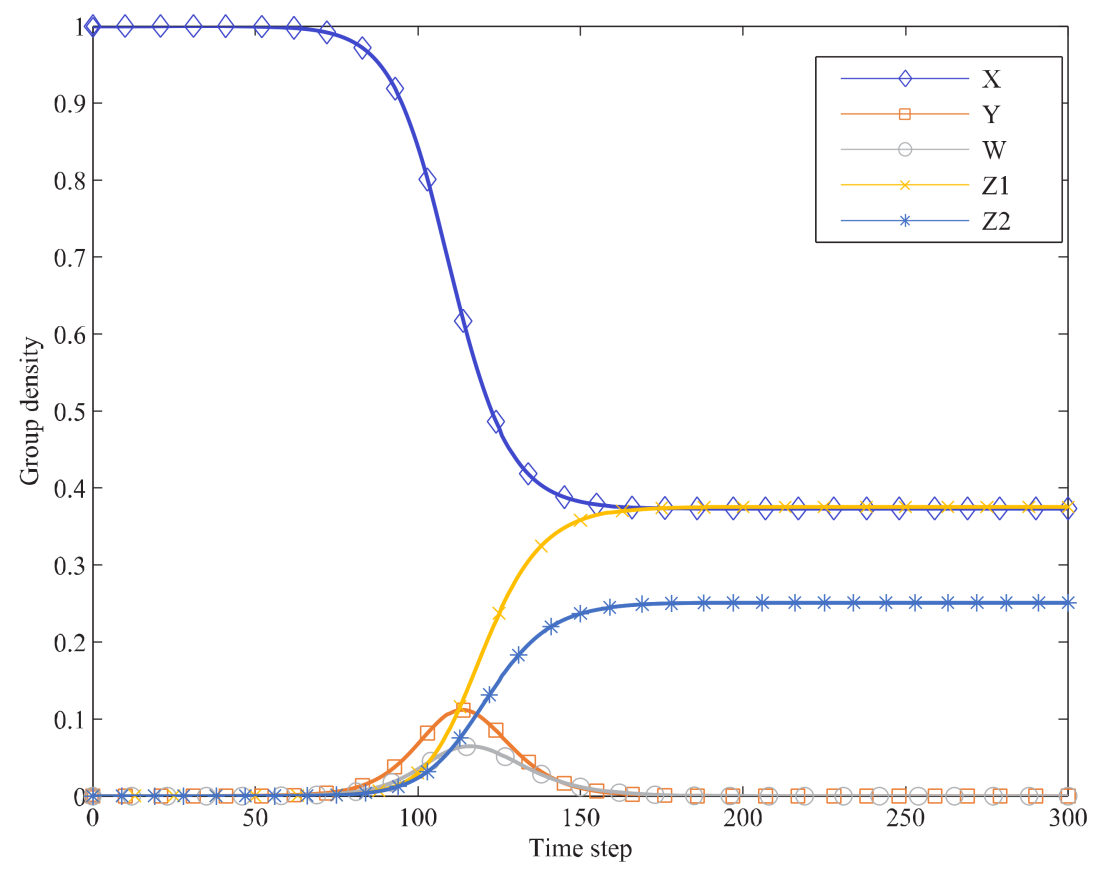

Figure 3. Group density curves for rumor propagation in a closed system XYWZ1Z2-C.

\subsection{Impact of Ignorant with Critical Ability on Rumor Propagation}

The proportion of the ignorant without critical ability was changed, i.e., $\varphi=0.9,0.7$, $0.5,0.3$, respectively; then the proportion of ignorant with a critical ability $(1-\varphi)$ was set as $0.1,0.3,0.5,0.7$, respectively, and the values of other parameters were listed in Table 1 . The group density curves of ignorants, spreaders, skeptics, stiflers who believed the rumor, and stiflers who did not believe the rumor were plotted under different values, as displayed in Figure 4.

As shown in Figure 4, the proportion of ignorant with critical ability $(1-\varphi)$ increased as $\varphi$ decreased. It could be concluded that: (1) when the rumor spreading ended, the reduced quantity in the population density of ignorants decreased, namely, the scale of rumor 
spreading narrowed down; (2) the peak of the population density of ignorants decreased, namely, the intensity of rumor propagation decayed; (3) the peak value of the skeptics' population density showed a trend of the first rose after declined, which was beneficial for identifying rumor information; (4) the duration of rumor spreading prolonged, and the process of rumor spreading gradually changed into the identification of rumor information; (5) when the spread of rumors ended, the population density of stiflers who believe the rumor decreased, namely, the negative influence of rumor spreading declined; (6) when rumor propagation ended, the population density of stiflers who did not believe the rumor first increased and then diminished, which was conducive to exert the function of the rumor refutation mechanism.

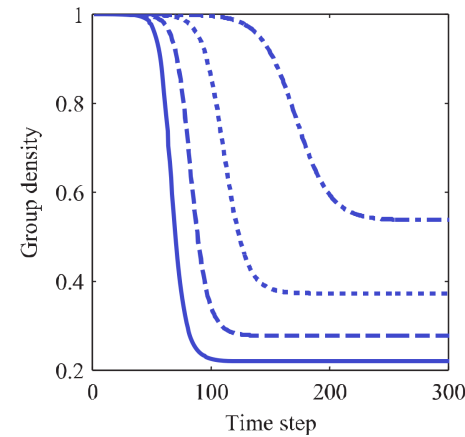

(a) $\mathrm{X}$

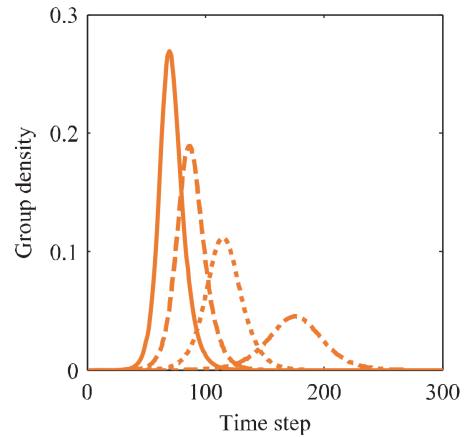

(b) $\mathrm{Y}$

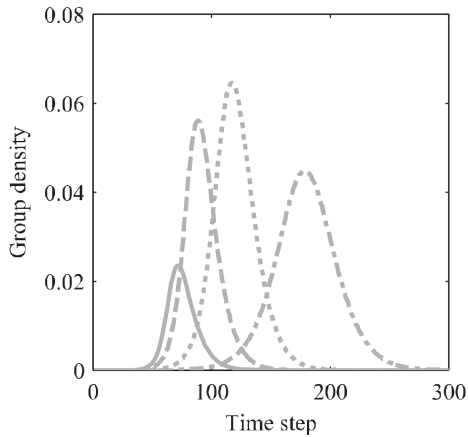

(c) W

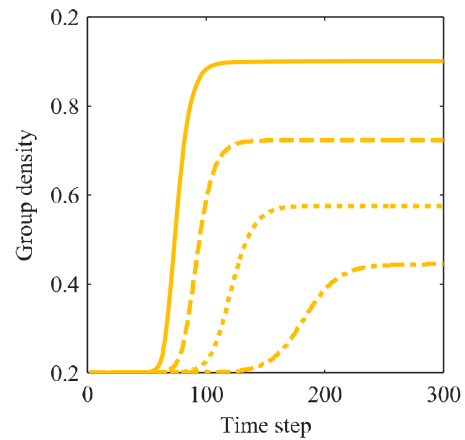

(d)Z1

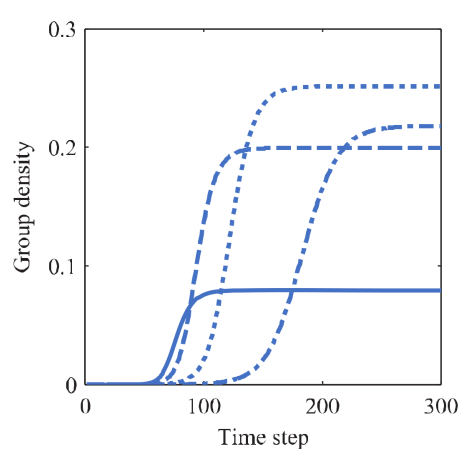

(e)Z2

Figure 4. Group density curves with $\varphi=0.9,0.7,0.5,0.3$ for $(\mathbf{a}) \mathrm{X} ;(\mathbf{b}) \mathrm{Y} ;$ (c) W; (d) Z1; (e) Z2. Note: The solid line was used for $\varphi=0.9$, the dashed line for $\varphi=0.7$, the dotted line for $\varphi=0.5$, and the dash-dotted line for $\varphi=0.3$.

\subsection{Impact of Rumor Refutation Mechanism on Rumor Propagation}

The strength of the rumor refutation mechanism was changed as $\lambda=0.05,0.1,0.2$, and 0.4 , respectively. The greater the value of $\lambda$, the stronger the feedback mechanism of stiflers who did not believe the rumor. To more profoundly perceive the impact of the strength of the rumor refutation mechanism on rumor propagation, the rumor propagation model described by Equation (1) was simulated. The optimized parameters were $\varphi=0.2, \alpha=0.2$, $\delta=0.2, \eta=0.1$, and $\theta=0.5$. The density curves of each group at different values of $\lambda$ are displayed in Figure 5.

As illustrated in Figure 5, as the rumor refutation mechanism strengthened: (1) at the end of rumor propagation, the proportion in the density of ignorants diminished, indicating that the scale of rumor propagation shrank; (2) the peak of the spreaders and the rumor spreading intensity decreased; (3) the peak of skeptics gradually decreased, indicating that the demand for rumor identification to control rumor propagation decreased; (4) the duration of rumor spreading shortened, which was beneficial in controlling the spread of rumors; (5) at the end of rumor spreading, the population density of stiflers who believed the rumor decreased, that is to say, the negative impact of rumor spreading decays; (6) at the end of rumor spreading, the population density of stiflers who did not believe the 
rumor decreased. In other words, the number of stiflers who did not believe the rumor needed to control rumor spreading was reduced.

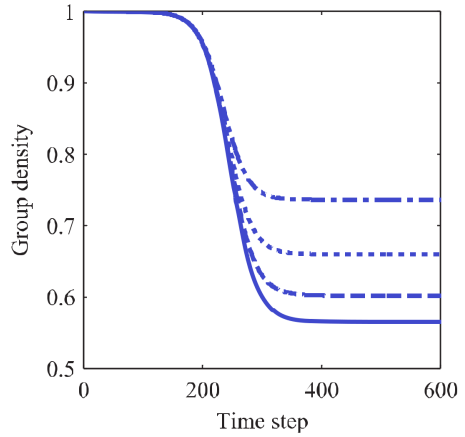

(a) $\mathrm{X}$

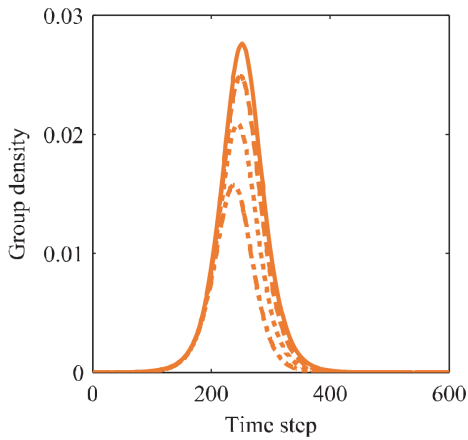

(b) $\mathrm{Y}$

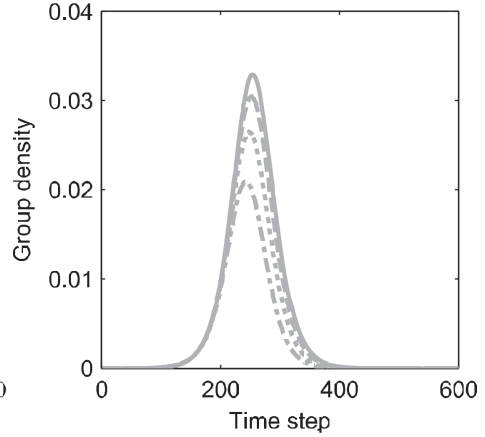

(c) W

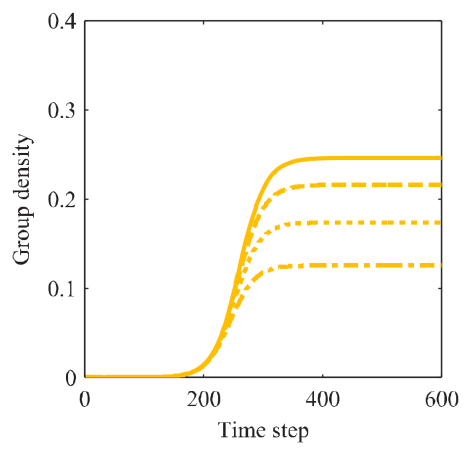

(d)Z1

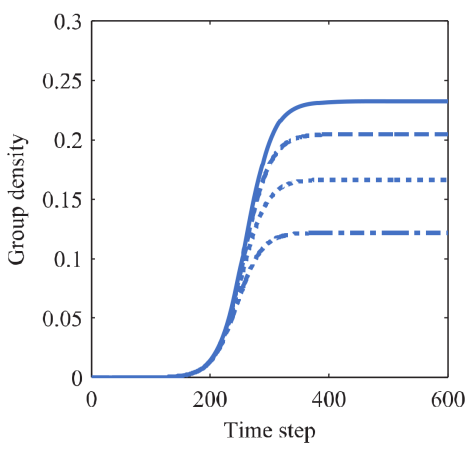

(e)Z2

Figure 5. Group density curves with $\lambda=0.05,0.1,0.2,0.4$ for (a) X; (b) $\mathrm{Y}$; (c) W; (d) Z1; (e) Z2. Note: the solid line was used for $\lambda=0.05$, the dashed line for $\lambda=0.1$ the dotted line for $\lambda=0.2$, and the dash-dotted line for $\lambda=0.4$.

\subsection{Impact of the Importance of Identifying Rumor on Rumor Propagation}

The identification of various rumors was of different importance. Individuals tended to be more cautious about rumors with higher identification importance, which increased the time required to identify rumors. Parameter $\eta$ could be used to distinguish rumor information with different identifying importance. The larger the $\eta$ value, the less important the identification of rumors. To apparently present the importance of rumor identification on rumor propagation, the numerical simulation was conducted, the optimized parameters were $\varphi=0.2, \alpha=0.2, \delta=0.2, \theta=0.5, \lambda=0.2$. Considering different identification importance of the rumor information, $\eta$ was set to be $0.01,0.1,0.99$, respectively. The corresponding group density curves are displayed in Figure 6.

Based on the above $\eta$ values, the rumor information was split into a rumor with an extremely high identification importance $(\eta=0.01)$, a rumor with a high identification importance $(\eta=0.1)$, and a rumor with a low identification importance $(\eta=0.99)$. As shown in Figure 6, with the increase in $\eta$, the identification importance of rumors decreased. Moreover, it could be concluded that: (1) at the end of the rumor propagation, the reduction in the group density of the ignorants decreased, indicating that the scale of rumor propagation shrank; (2) the peak of the spreaders decreased gradually; that is, the intensity of the rumor spreading decreased; (3) the peak of skeptics decreased gradually; that is, the pressure on the demand for true information gradually relieved; (4) the duration of rumor spreading shortened; that is, rumor spreading tended to subside; (5) when rumor propagation ended, the density of stiflers who believed the rumor decreased; that is, the negative impact of rumor spreading reduced; (6) at the end of rumor spreading, the density of stiflers who did not believe the rumor decreased, namely, the number of stiflers who did not believe the rumor required to control the spread of rumors was reduced; (7) if the situation of rumor information was within the range of a higher identification importance, the importance of 
identifying rumors had a greater impact on the rumor propagation process. On the contrary, the influence became smaller gradually while the rumor information was in the range of lower identification importance. Consequently, in emergency management, priority should be given to identifying rumor information in a range of higher identification importance, thereby enhancing the effect of rumor control management.

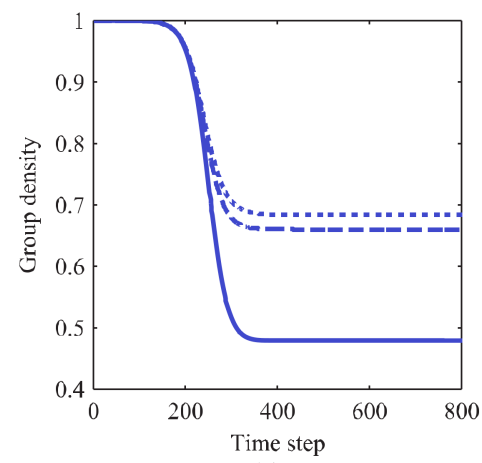

(a) $\mathrm{X}$

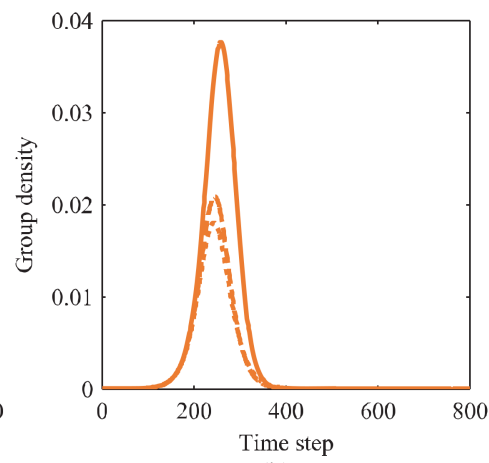

(b) $\mathrm{Y}$

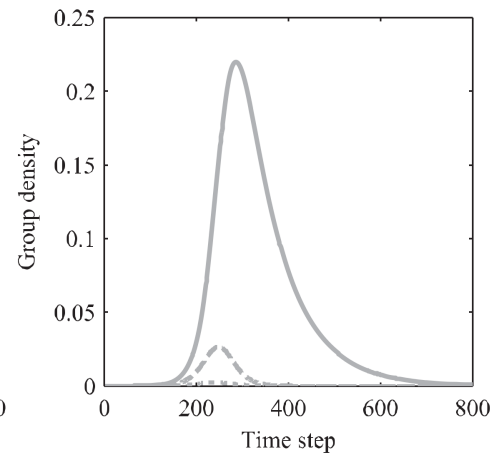

(c) W

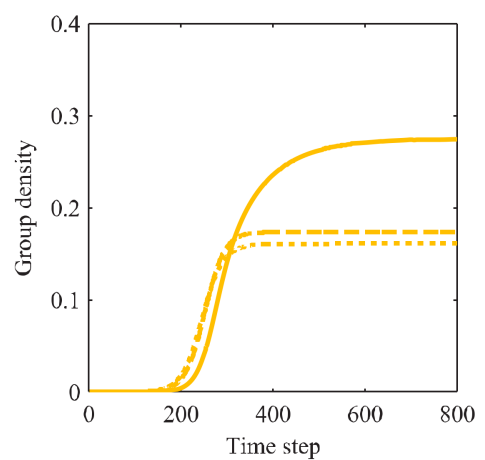

(d) Z1

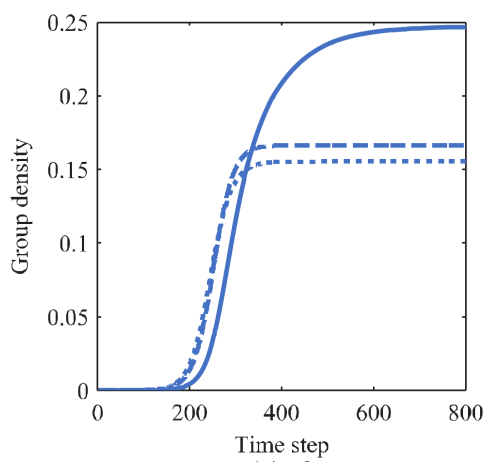

(e)Z2

Figure 6. Group density curves with $\eta=0.01,0.1,0.99$ for (a) X; (b) Y; (c) W; (d) Z1; (e) Z2. Note: solid line was used for $\eta=0.01$; dashed line for $\eta=0.1$; dotted line for $\eta=0.99$.

\subsection{Impact of Skeptics' Ability to Identify Rumors on Rumor Propagation}

Skeptics actively obtained evidence of falsifying rumor information, namely, that rumor refutation information. If skeptics could not obtain the refutation information over an expected period, they would abandon the attempt to attain refutation information. Consequently, skeptics were converted into stiflers who believed the rumor. On the contrary, if skeptics could obtain refutation information in the expected time, they turned into stiflers who did not believe the rumor. The possibility of the skeptics obtaining the refutation information indicated the skeptics' capacity to identify rumors. The smaller the parameter $\theta$, the larger the $(1-\theta)$, the higher the skeptics' ability to identify rumors. To reveal the impact of the skeptics' ability to identify rumors on the process of rumor propagation, the numerical simulation was conducted. $\theta=0.9,0.6,0.3,0.1,(1-\theta)=0.1$, $0.4,0.7$, and 0.9 , respectively. The other optimized parameters were $\varphi=0.2, \alpha=0.2, \delta=0.1$, $\lambda=0.2, \eta=0.1$. The group density curves are displayed in Figure 7 .

As shown in Figure 7, as the skeptics' ability to identify rumors enhanced, it could be concluded that: (1) at the end of the rumor propagation, the reduction in the group density of ignorants decreased; that is, the scale of rumor propagation decreased; (2) with the peak of spreaders gradually decreasing, the intensity of rumor spreading declined; (3) the peak of skeptics gradually decreased; thus, the pressure on the demand for true information was gradually lightened; (4) at the end of rumor propagation, the density of stiflers who believed the rumor decreased; that is, the negative impact of rumor spreading diminished; (5) at the end of rumor propagation, the density of stiflers who did not believe the rumor was significantly magnified, which was in favor of stabilizing public sentiment. 


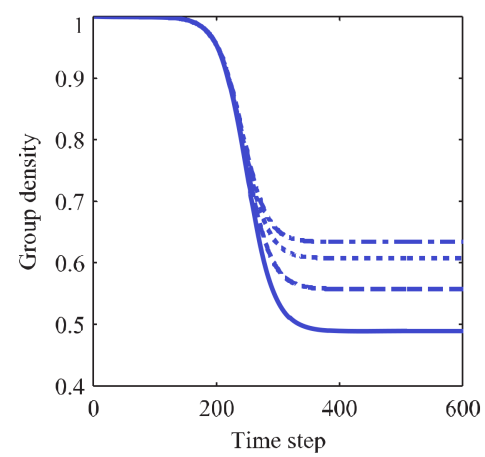

(a) $\mathrm{X}$

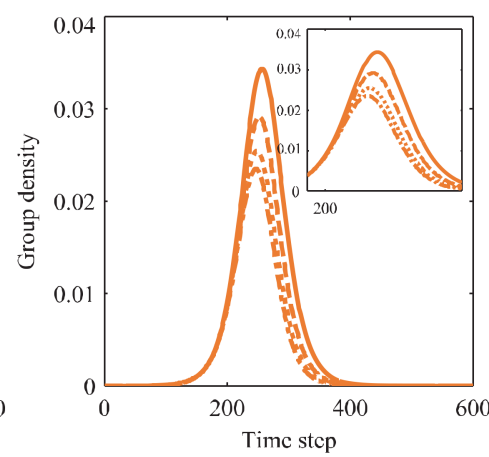

(b) $\mathrm{Y}$

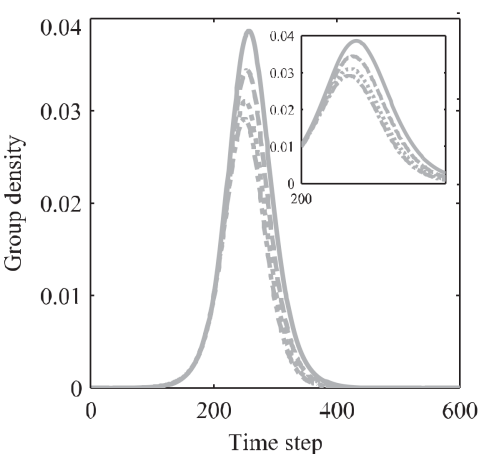

(c) W

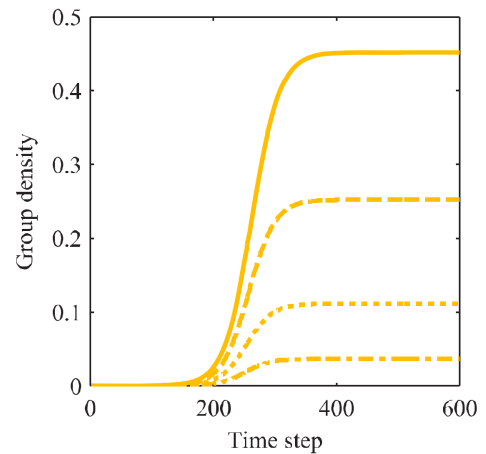

(d)Z1

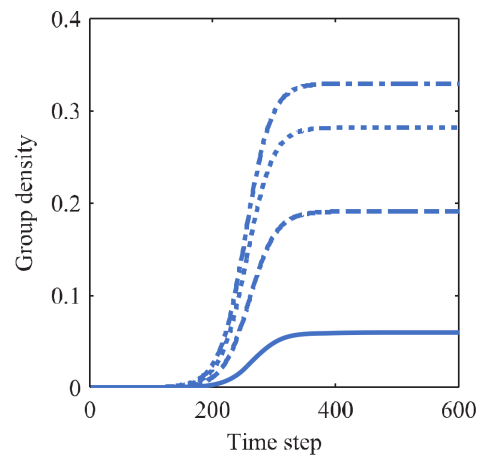

(e)Z2

Figure 7. Group density curves with $(1-\theta)=0.1,0.4,0.7,0.9$ for $(\mathbf{a}) \mathrm{X}$; (b) $\mathrm{Y} ;$ (c) W; (d) Z1; (e) Z2. Note: The solid line was used for $(1-\theta)=0.1$; the dashed line for $(1-\theta)=0.4$; dotted line for $(1-\theta)=0.7$; the dash-dotted line for $(1-\theta)=0.9$.

The single-factor analysis results for the closed rumor propagation system above demonstrated that the negative impact of rumor propagation could be reduced by enlarging the proportion of ignorants with critical ability in the system, enhancing the rumor refutation information mechanism, and improving the capacity of skeptics to identify rumors. The analysis of the importance of identifying rumors points out that rumors with high identification importance should be prior processed in emergency management, so as to minimize the negative impact of rumor propagation to the greatest extent. Therefore, the following measures can be taken to reduce the negative impact of rumor propagation as far as possible in the absence of official refutation information: (1) improve the media literacy of the public to enhance the ability to identify rumors; (2) raise the public's feedback awareness of rumor information; (3) provide open and effective information query and exchange platform; (4) popularize knowledge education related to emergencies. Concerning the rumor spreading in the specific network, compulsory immunization measures can be taken, such as deleting the rumor information released by the spreaders and promptly disseminating the refutation information issued by stiflers who do not believe the rumor, to directly control the rapid spread of rumors.

In the actual rumor spreading process, multiple influencing factors are prone to engender cross-impact, and the rumor propagation system is also susceptible to other external factors. For instance, for rumors with strong negative emotions, increasing the proportion of ignorants with critical ability will increase the number of skeptics in the system, which may bring about an increase in the demand for true information. The strength of the refutation information is affected by official accountability. Hence, under the specific context of rumor spreading, it is necessary to consider the characteristics of influencing factors in actual situations and select reasonable measures accordingly to control rumor spreading. 


\section{Analysis of Rumor Propagation Rules in an Open System}

According to the analysis of the open rumor spreading system in Section 2.2, a threshold $\mathrm{R}_{0}=\left(\varphi^{*} \alpha\right)$ exists in the process of rumor spreading. If and only if $\mathrm{R}_{0}>1$, i.e., $\varphi>\mu / \alpha$, the rumor propagation system has a rumor-endemic equilibrium point. In other words, when the proportion of ignorants without critical ability in the system is greater than a certain extent, rumors can continue spreading stably in the open rumor spreading system. Let the initial total population be $N=10^{6}$. When a spreader appears in the system, i.e., $Y=1$, then $X=N-1 \approx 10^{6}, W=0, Z_{1}=0, Z_{2}=0$. When the immigration rate is equal to the emigration rate, the total population does not change in the rumor spreading process. Next, the four-order Runge-Kutta method was utilized to solve the differential Equation (5) to simulate and analyze the rumor propagation development process under the impact of population migration.

\subsection{Analysis of the General Rules of Rumor Propagation in an Open System}

Let $\kappa=\mu=0.005$ and $R_{0}>1$, the remaining parameters are defined as Table 1 . The variation trend in the density of each group was simulated and analyzed. In addition, the rumor spreading process was compared with that of the closed rumor spreading system under the same conditions, as shown in Figure 8.

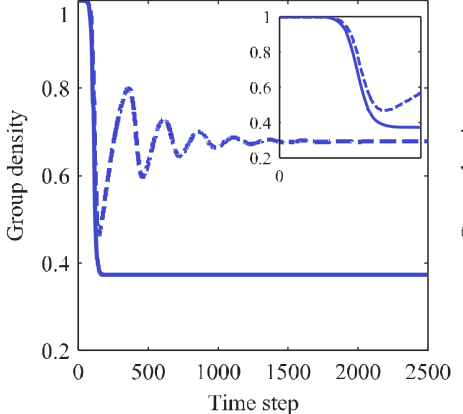

(a) $\mathrm{X}$

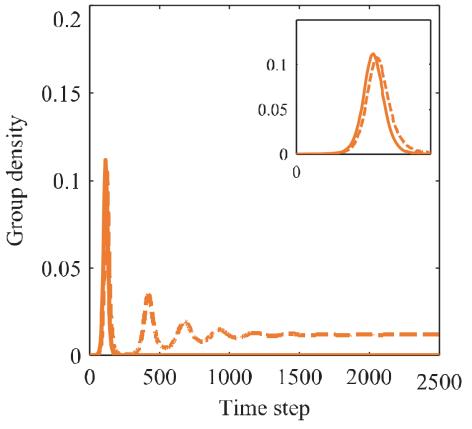

(b) $\mathrm{Y}$

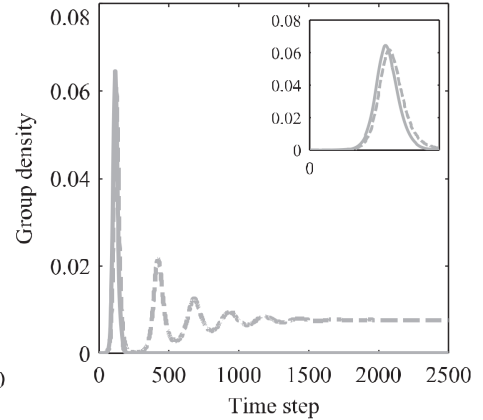

(c) W

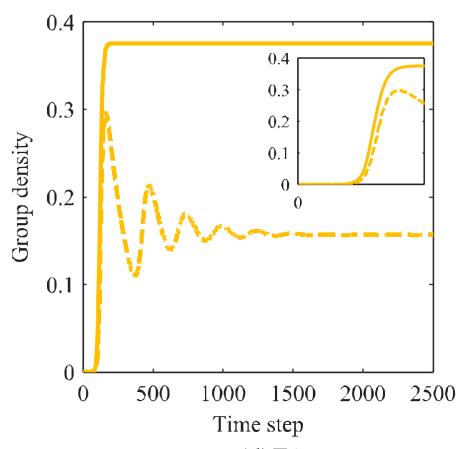

(d)Z1

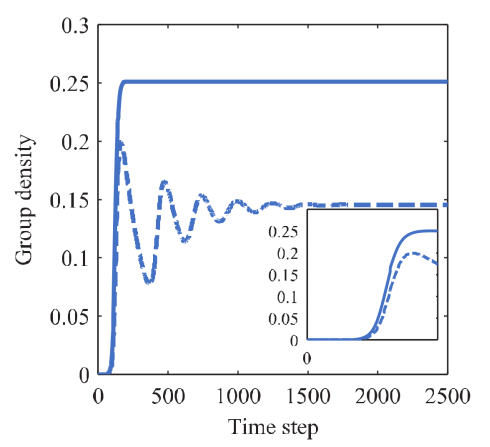

(e)Z2

Figure 8. Comparative analysis of rumor propagation general rules between open system XYWZ1Z2-O and closed system XYWZ1Z2-C for (a) X; (b) Y; (c) W; (d) Z1; (e) Z2. Note: The solid line was used for $\kappa=\mu=0$; dashed line for $\kappa=\mu=0.005$.

When the population migrated to the open rumor spreading system, the rumor spreading could exceed the threshold value. As shown in Figure 8, it could be concluded that: (1) the rumor spreading process presented a fluctuating development, the fluctuation range gradually reduced, and, finally, maintained a dynamic rumor spreading equilibrium state. It was evident that the rumor propagation system had a rumor-endemic equilibrium point. Contrary to the closed rumor spreading process, in the open system, the rumor did not disappear in this autonomous system. Therefore, in the management of the rumor spreading in the open system, countermeasures should be taken to prevent the continuous long-term negative impact on the system. (2) When rumor spreading reached the final equilibrium state, the reduction in the density of ignorants, the density of stiflers 
who believed the rumor, and stiflers who did not believe the rumor in the open system were all decreased compared with those in a closed system. Hence, in the long run, the negative impact of rumor propagation could be minimized by population immigration and emigration.

\subsection{Analysis on the Rumor Spreading Rules at the Critical Point}

Let $\kappa=\mu=0.04$, and the proportion of ignorants without critical ability in the rumor spreading system $\varphi=0.19,0.2,0.21$ corresponding to $R_{0}<1, R_{0}=1, R_{0}>1$. The other parameters were set as shown in Table 1 . To compare and analyze the rumor propagation process at the critical point, the rumor propagation mean-field of Equation (5) was solved, and the developing trend of the density of each group at the initial stage of rumor propagation was plotted, as displayed in Figure 9.

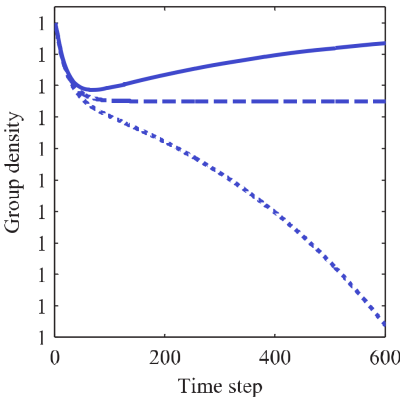

(a)X

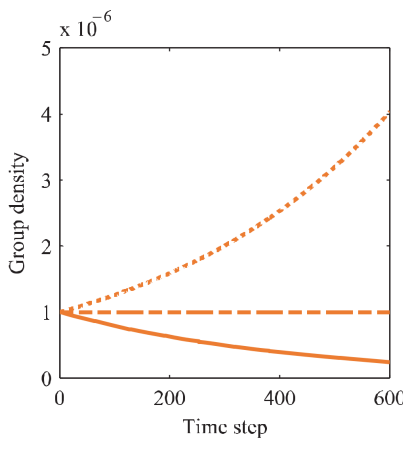

(b) Y

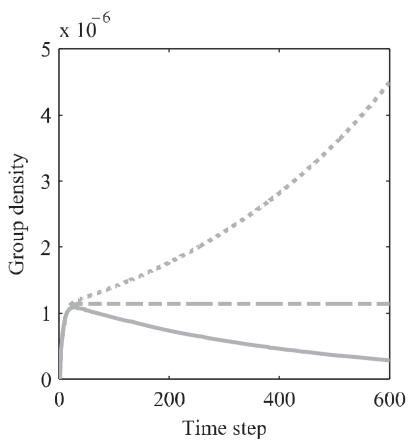

(c) W

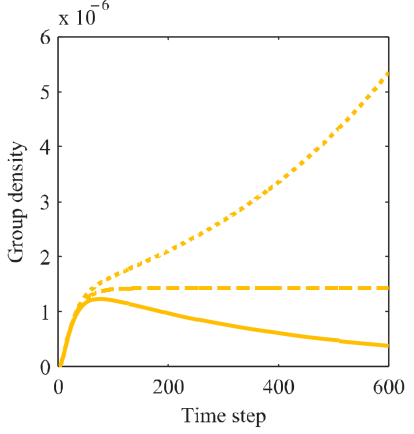

(d)Z1

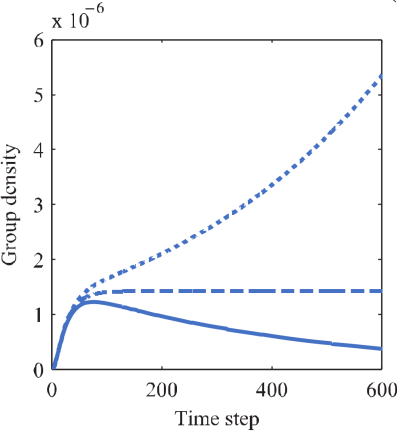

(e)Z2

Figure 9. Rumor propagation rules analysis at a critical point in an open system XYWZ1Z2-O for (a) X; (b) Y; (c) W; (d) Z1; (e) Z2. Note: solid line was used for $\kappa=\mu=0.04, \varphi=0.19$ and $\mathrm{R}_{0}<1$, dashed line for $\kappa=\mu=0.04, \varphi=0.2, R_{0}=1$, and dotted line for $\kappa=\mu=0.04, \varphi=0.21, R_{0}>1$.

As shown in Figure 9, the initial development process of rumor propagation was quite different at the critical point. At the critical point $\left(R_{0}=1\right)$, each group in the system remained basically unchanged, namely, the rumor information has not spread. When the rumor propagation system failed to reach the critical point $\left(R_{0}<1\right)$, the population density of spreaders expressed a downward trend, namely, that rumors could not widely disseminate. When the rumor propagation system came to the critical point above $\left(R_{0}>1\right)$, the population density of spreaders signified an upward trend, and rumors could spread. As a result, the rumor spreading system had a threshold point for the mutation of rumor transmission law, which was consistent with the results of the analysis in Section 2.2.

In the actual rumor spreading system, the population immigration and emigration rate was usually petty, and the proportion of ignorants without critical ability was generally large; thus, the rumor spreading could exceed the critical threshold effortlessly. As a result, rumor information could continue to spread stably, which is slightly affected by the small population immigration and emigration rate. Therefore, it was necessary to take active measures to control the long-term spread of rumors or reduce the proportion of characters 
excluding critical ability in the system, so as to reduce the possibility of an extensive spread of rumors fundamentally.

\subsection{Impact of Immigration and Emigration Rate on Rumor Propagation}

To analyze the impact of the change in the immigration and emigration rate on the results of rumor propagation, $\kappa=\mu=0.005$ and $\kappa=\mu=0.01$ was taken, respectively. At the same time, $\varphi>\mu / \alpha$ and $\mathrm{R}_{0}>1$ hold. The other parameter settings are listed in Table 1 . The comparative analysis of the rumor spreading in the above two cases is shown in Figure 10.

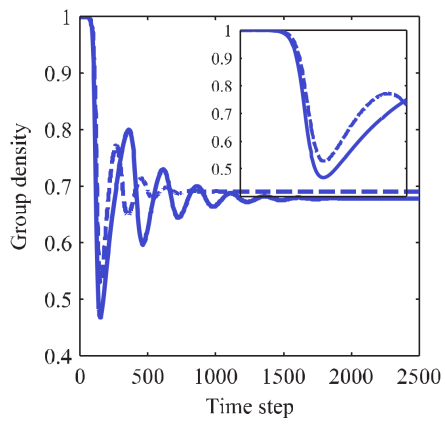

(a) $\mathrm{X}$

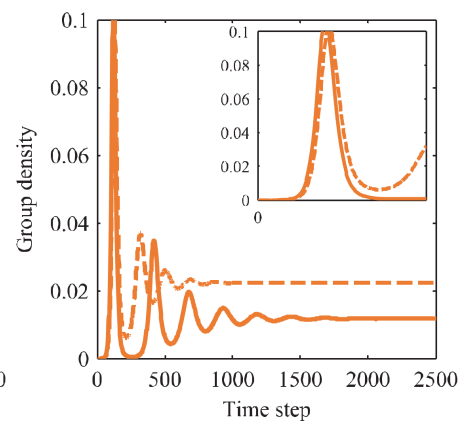

(b) $\mathrm{Y}$

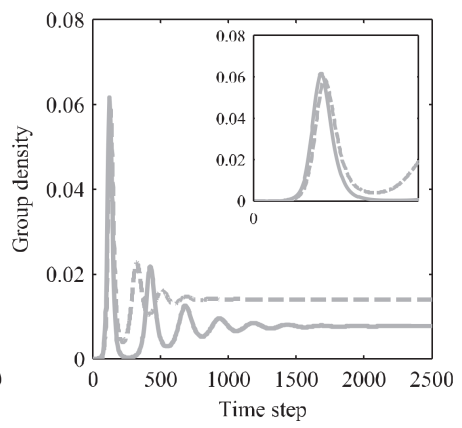

(c) W

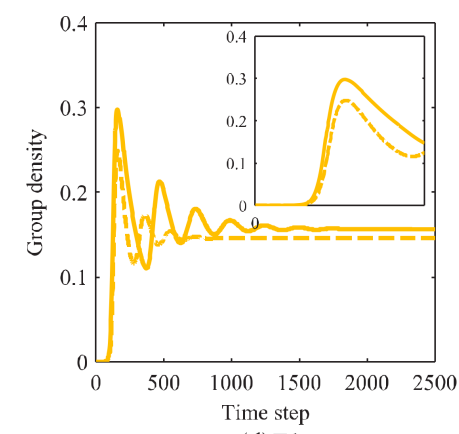

(d)Z1

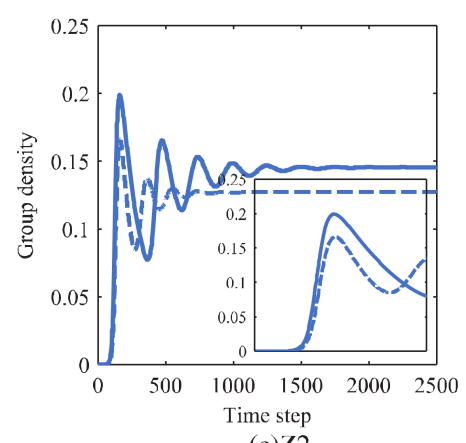

(e) $Z 2$

Figure 10. Impact analysis of immigration and emigration rate for rumor propagation in an open system XYWZ1Z2-O for (a) X; (b) Y; (c) W; (d) Z1; (e) Z2. Note: The solid line was used for $\kappa=\mu=0.005$; dashed line for $\kappa=\mu=0.01$.

As shown in Figure 10, when the immigration and emigration rates increased, the fluctuation range of rumor propagation decreased, and the fluctuation time decreased. When the rumor reached the equilibrium state, the densities of ignorants, spreaders, and skeptics in the system increased, while the densities of stiflers who believed the rumor and stiflers who did not believe the rumor decreased. Therefore, it was imperative to strengthen the rumor management in the rumor propagation to prevent the negative impact of the continuous spread of rumors on the system when the immigration and emigration rates increased. In rumor management, the characteristics of the rumor spreading system should be fully considered and reasonable countermeasures should be formulated in combination with the development rules of rumor spreading.

\section{Discussion and Conclusions}

\subsection{Discussion about the Application}

From the above analysis, we could find that the development process of rumor dissemination could be divided into four stages. It would make a significant contribution to reducing the negative effect of rumor propagation. Accordingly, one strategy may be proposed to reduce the losses brought by rumor propagation combined with artificial intelligence technology. When an emergency event happens, rumors also appear. The authority should determine the values of the parameters in the rumor propagation model. Subsequently, the authority can obtain the estimated population of ignorants, spreaders, skeptics, stiflers who believe the rumor, and stiflers who do not believe the rumor in 
different stages. Additionally, the authority can also obtain the possible development of a rumor. Then, according to the number of views, comments, and comment content of rumor information on social media, such as Twitter and Weibo, the authority can also obtain the real population in five groups. Thus, the authority may evaluate the actual situations and take appropriate measures accordingly. The discussion above is only a general framework. There are still many details to be refined and much work to be performed in future.

\subsection{Conclusions}

In this paper, we established a rumor propagation model based on the rumor refutation mechanism. In this model, the roles of individuals in controlling rumor spreading and the individuals' behavioral regularities of rumor spreading were fully considered in both the closed system and the open system. The equilibrium points in both the closed system and open system were discussed. The rumor spreading in the closed system was autonomous, and the spreading process was deterministic. While in the open system, the critical threshold existed in the open rumor propagation system. In addition to the theoretical analysis, several numerical simulations were performed in both the closed system and open system. We selected appropriate parameters for Equations (1) and (5) to conduct a simulation to verify the correctness of the theories analysis. Additionally, it was found that the development process of rumor dissemination could be divided into four stages: latent period, progressive period, intense period, and recession period. It was helpful for the authorities to take measures at the appropriate time. We also analyzed the rumor propagation law under the different influencing factors. Some measures, such as providing open and efficient information queries and exchange platforms by the authorities, could be taken to stop the propagation of rumors. Under the impact of population migration, rumor spreading could exceed the threshold, and the rumor spreading process in an open system presented a fluctuating development. The rumor did not disappear in this autonomous system. Moreover, the negative impact of rumor propagation could be minimized by population immigration and emigration. Furthermore, spreaders were required for the dynamic equilibrium state of rumor propagation and we should notice that the number of rumor spreaders increased with the increase in the population migration rate.

In this article, the law of rumor propagation based on the mean-field equation was primarily studied. However, the impact of the actual network structure on rumor dissemination was not considered. Moreover, the rumor spreading process was influenced by many factors inside and outside the system with a complicated influencing mechanism. When formulating the control measures of rumor propagation in emergent events, it is necessary to combine the characteristics of the actual rumor propagation system and take comprehensive optimization measures to effectively reduce the negative impact of rumor propagation and control rumor propagation.

Author Contributions: Conceptualization, J.C. and Q.S.; methodology, J.C.; software, C.C. and L.D.; formal analysis, J.C., C.C., Q.S. and Y.Z.; investigation, C.C., Y.Z. and R.X.; writing-original draft preparation, C.C. and Q.S.; writing-review \& editing, S.Y. and C.C.; visualization, L.D. and R.X.; supervision, S.Y.; project administration, S.Y.; funding acquisition, J.C. and Y. Z. All authors have read and agreed to the published version of the manuscript.

Funding: This research was funded by the National Natural Science Foundation of China (NSFC) under grant no. 51374242 and the Graduated Students' Research and Innovation Fund Project of Central South University under grant no. 2021zzts0283.

Data Availability Statement: The data used to support the findings of this study are included within the article.

Acknowledgments: The authors would like to thank all the participants of this study and the authors also gratefully acknowledge the anonymous reviewers, who were instrumental in improving an earlier version of this article.

Conflicts of Interest: The authors declare no conflict of interest. 


\section{References}

1. Daley, D.J.; Kendall, D.G. Stochastic rumours. J. Inst. Math. Its Appl. 1965, 1, 42-55. [CrossRef]

2. Maki, D.P.; Thompson, M. Mathematical Models and Applications: With Emphasis on the Social, Life, and Management Sciences; Prentice-Hall: Princeton, NJ, USA, 1973.

3. Huo, L.-A.; Chen, S.; Zhao, L.-J. Dynamic analysis of the rumor propagation model with consideration of the wise man and social reinforcement. Phys. A: Stat. Mech. Its Appl. 2021, 571, 125828. [CrossRef]

4. Yang, S.; Jiang, H.-J.; Hu, C.; Yu, J.; Li, J.-R. Dynamics of the rumor-spreading model with hesitation mechanism in heterogenous networks and bilingual environment. Adv. Differ. Equations 2020, 2020, 628. [CrossRef]

5. Zhu, L.-H.; Huang, X.-Y.; Liu, Y.; Zhang, Z.-D. Spatiotemporal dynamics analysis and optimal control method for an SI reactiondiffusion propagation model. J. Math. Anal. Appl. 2021, 493, 124539. [CrossRef]

6. Abta, A.; Laarabi, H.; Rachik, M.; Alaoui, H.T.; Boutayeb, S. Optimal control of a delayed rumor propagation model with saturated control functions and $\mathrm{L}^{\wedge}(1-)$ type objectives. Soc. Netw. Anal. Min. 2020, 10, 1-15. [CrossRef]

7. Laarabi, H.; Abta, A.; Rachik, M.; Bouyaghroumni, J. Stability analysis of a delayed rumor propagation model. Differ. Equ. Dyn. Syst. 2016, 24, 407-415. [CrossRef]

8. Huo, L.-A.; Huang, P.-Q.; Guo, C.-X. Analyzing the dynamics of a rumor transmission model with incubation. Discret. Dyn. Nat. Soc. 2012, 328151. [CrossRef]

9. Jain, A.; Dhar, J.; Gupta, V. Rumor model on homogeneous social network incorporating delay in expert intervention and government action. Commun. Nonlinear Sci. 2020, 84, 105189. [CrossRef]

10. Wang, H.; Deng, L.; Huang, Y.-S.; Zhao, S. A variant epidemic propagation model suitable for rumor spreading in online social network. In Proceedings of the International Conference on Machine Learning \& Cybernetics, Xi'an, China, 15-17 July 2012; pp. 1258-1262. [CrossRef]

11. Zan, Y.-L.; Wu, J.-J.; Li, P.; Yu, Q.-L. SICR rumor spreading model in complex networks: Counterattack and self-resistance. Phys. A Stat. Mech. Its Appl. 2014, 405, 159-170. [CrossRef]

12. Xia, L.-L.; Jiang, G.-P.; Song, B.; Song, Y.R. Rumor spreading model considering hesitating mechanism in complex social networks. Phys. A Stat. Mech. Its Appl. 2015, 437, 295-303. [CrossRef]

13. Fu, M.-L.; Feng, J.; Lande, D.; Dmytrenko, O.; Manko, D.; Prakapovich, R. Dynamic model with super spreaders and lurker users for preferential information propagation analysis. Phys. A Stat. Mech. Its Appl. 2021, 561, 125266. [CrossRef]

14. Huo, L.A.; Lin, T.; Huang, P. Dynamical behavior of a rumor transmission model with psychological effect in emergency event. Abstr. Appl. Anal. 2013, 4339-4344. [CrossRef]

15. Nekovee, M.; Moreno, Y.; Bianconi, G.; Marsili, M. Theory of rumour spreading in complex social networks. Phys. A Stat. Mech. Its Appl. 2008, 374, 457-470. [CrossRef]

16. Kawachi, K.; Seki, M.; Yoshida, H.; Otake, Y.; Warashina, K.; Ueda, H. A rumor transmission model with various contact interactions. J. Theor. Biol. 2008, 253, 55-60. [CrossRef] [PubMed]

17. Zhao, L.-J.; Wang, Q.; Cheng, J.-J.; Chen, Y.C.; Wang, J.-J.; Huang, W. Rumor spreading model with consideration of forgetting mechanism: A case of online blogging LiveJournal. Phys. A Stat. Mech. Its Appl. 2011, 390, 2619-2625. [CrossRef]

18. Wang, Y.-Q.; Yang, X.-Y.; Han, Y.-L.; Wang, X.-A. Rumor spreading model with trust mechanism in complex social networks. Commun. Theor. Phys. 2013, 59, 510-516. [CrossRef]

19. Qiu, L.-Q.; Liu, S.-Q. C-SIW rumor propagation model with variable propagation rate and perception mechanism in social networks. Discret. Dyn. Nat. Soc. 2020, 5712968. [CrossRef]

20. Afassinou, K. Analysis of the impact of education rate on the rumor spreading mechanism. Phys. A Stat. Mech. Its Appl. 2014, 414, 43-52. [CrossRef]

21. Hui, H.-W.; Zhou, C.-C.; Lu, X.; Li, J.-R. Spread mechanism and control strategy of social network rumors under the influence of COVID-19. Nonlinear Dyn. 2020, 101, 1933-1949. [CrossRef]

22. Hosni, A.I.E.; Li, K.; Ahmad, S. Analysis of the impact of online social networks addiction on the propagation of rumors. Phys. A Stat. Mech. Its Appl. 2020, 542, 123456. [CrossRef]

23. Chen, X.-L.; Wang, N. Rumor spreading model considering rumor credibility, correlation and crowd classification based on personality. Sci. Rep. 2020, 10, 5887. [CrossRef]

24. Srinivasan, S.; Babu, D.L.D. A social immunity based approach to suppress rumors in online social networks. Int. J. Mach. Learn. Cybern. 2021, 12, 1281-1296. [CrossRef]

25. Zhang, Z.-L.; Zhang, Z.-Q. An interplay model for rumour spreading and emergency development. Phys. A Stat. Mech. Its Appl. 2009, 388, 4159-4166. [CrossRef]

26. Huo, L.-A.; Huang, P.-Q.; Fang, X. An interplay model for authorities' actions and rumor spreading in emergency event. Phys. A Stat. Mech. Its Appl. 2011, 390, 3267-3274. [CrossRef]

27. Zhao, L.-J.; Wang, Q.; Cheng, J.-J.; Zhang, D.; Ma, T.; Chen, Y.-C.; Wang, J.-J. The impact of authorities' media and rumor dissemination on the evolution of emergency. Phys. A Stat. Mech. Its Appl. 2012, 391, 3978-3987. [CrossRef]

28. Zhao, L.-J.; Wang, X.-L.; Qiu, X.-Y.; Wang, J.-J. A model for the spread of rumors in Barrat-Barthelemy-Vespignani (BBV) networks. Phys. A Stat. Mech. Its Appl. 2013, 392, 5542-5551. [CrossRef]

29. Zhao, L.-J.; Wang, J.-J.; Chen, Y.-C.; Wang, Q.; Cheng, J.-J.; Cui, H.-X. SIHR rumor spreading model in social networks. Phys. A Stat. Mech. Its Appl. 2012, 391, 2444-2453. [CrossRef] 